Running head: Self-Prioritization in Vision, Audition, and Touch

\title{
Self-Prioritization in Vision, Audition, and Touch
}

\author{
Sarah Schäfer $^{* 1}$, Ann-Katrin Wesslein ${ }^{* 12}$, Charles Spence ${ }^{2}$, \\ Dirk Wentura ${ }^{3}, \&$ Christian Frings ${ }^{1}$ \\ ${ }^{1}$ University of Trier, Universitätsring 15, 54296 Trier, Germany \\ ${ }^{2}$ University of Oxford, 9 South Parks Road, Oxford, OX1 3UD, UK \\ ${ }^{3}$ Saarland University, Campus A2 4, 66123 Saarbrücken, Germany \\ *shared first authorship
}

\section{Acknowledgements:}

We would like to thank Karina Katarzyna Czech, Fee Carolin Gierens, Benedikt Graf, Ira Katrin Gröne, Jule Heckmann, Miriam Hehlmann, Tabea Milena Henn, Esther Karst, Barbara Lenz, and Julia Strojny from the University of Trier for collecting the data.

Corresponding author:

Sarah Schäfer

Department of Psychology, Cognitive Psychology, University of Trier schaefers@uni-trier.de Phone: +49 (0)651 2012073 Fax +49(0)6512012955 


\begin{abstract}
To investigate self-prioritization independently of stimulus familiarity, Sui, He, and Humphreys (2012) introduced a new paradigm in which different geometric shapes are arbitrarily associated with self-relevant (e.g., "I") and neutral labels (e.g., "stranger"). It has now been repeatedly demonstrated that in a subsequently-presented matching task this association leads to faster and more accurate verifications of self-relevant shape-label pairings than neutral shape-label pairings. In order to assess whether this self-prioritization effect represents a general selection mechanism in human information processing, we examined whether it is limited to the visual modality. Therefore, besides visual stimuli, auditory and vibrotactile stimuli were also associated either to self-relevant or to neutral labels. The findings demonstrate that self-prioritization represents a general tendency influencing human information processing, one that operates across the senses. Our results also highlight a topdown component to self-prioritization.
\end{abstract}

Keywords: Self-prioritization; selection mechanism; vision; audition; touch 
Environmental information from different sources reaches our senses simultaneously.

From this large amount of multisensory information, it is important to select just that information that is currently relevant, and to ignore that which is currently irrelevant. Selfrelevance is an important feature that predestines a piece of information to be selected for further processing. Diverse findings show that self-relevant information bypasses attentional thresholds more easily than information that is not linked to the self (e.g., Bargh 1982; Moray, 1959, for dichotic listening; Wuillemin and Richardson 1982, for the importance of expectation; Giesbrecht et al. 2009, for visual attentional blindness; Yang et al. 2013, for effects in visual search) and that attention is automatically captured and directed by information that is self-relevant (Alexopoulos et al. 2012).

So far in the field of self-prioritization research, self-relevant information has mostly consisted of the participant's own name, a picture of his or her face, or autobiographical information. Thus, the self-relevant stimuli typically used in these studies tend to have been highly overlearned and familiar and are typically compared to non-self-relevant stimuli which are not overlearned or nearly as familiar as the self-relevant ones. In order to avoid the confound of familiarity and self-relevance, Sui, He, and Humphreys (2012) introduced a paradigm in which, through training, simple geometric shapes are associated with the self, a familiar person, or a neutral stimulus by presenting the shape-label mappings on the screen (e.g., "[the participant's best friend's name] is a circle. You are a triangle. And a stranger is represented by a square.”). After this association phase, performance in self-relevant trials was enhanced as compared to performance in other-relevant trials (i.e., the participant's best friend and a stranger in the above example) thus demonstrating self-prioritization. In detail, after the association phase, participants performed a matching task in which they had to judge whether or not presented shape-label pairings (i.e., the label "you" presented with a triangle) were correct according to the learned associations. The results showed that verifications of 
correct self-relevant trials were performed faster and more accurately than verifications of

correct other-relevant trials, thus reflecting the self-prioritization effect (SPE).

Neurological investigations of the SPE using functional magnetic resonance imaging (fMRI) have revealed activation in separate brain regions for self-relevant (the ventral medial prefrontal cortex [vmPFC] and the left posterior superior temporal sulcus [LpSTS]) and nonself-relevant content (the dorsal frontoparietal control network), and a correlation of the SPE with a coupling of vmPFC and LpSTS (Sui et al. 2013b). Moreover, partially overlapping brain activations while suppressing self-associated or perceptually salient distractors, respectively, indicate that newly-acquired self-assignments affect neural activation comparably (though that is not to say equivalently) to perceptual salience (Sui et al. 2013a).

To date, the SPE has been replicated several times in visual matching tasks and has been generalized to different labels (see Sui et al. 2012, for findings concerning the SPE's independence of word length, frequency, and familiarity; Sui et al. 2013b; e.g., Schäfer et al. 2015). Furthermore, the effect has been extended from perception to action. For this purpose, simple movement directions (i.e., up, down, left, right) were assigned to the participant's self, to his/her mother, to a stranger, or to a neutral label; in the following trials, participants executed a movement (triggered by a cue) which was followed by a label. The participants then had to judge whether the particular movement-label pairing matched or not. Comparing performance in matching trials, an SPE was observed: That is, responses toward self-relevant movement-label pairings were faster and more accurate than responses toward other-relevant movement-label pairings (Frings and Wentura 2014).

According to the extension of the SPE from visual perception to action, one could expect an influence of self-relevance on information processing in modalities other than vision. In particular, there is a rich body of research dealing with the question of what we consider to be our self and how the tactile sense defines the border of the self (Gallace and Spence 2014). Many studies dealing with the so-called "full-body illusion" indicate 
somatosensory sensations as a relevant factor to discriminate our self from the environment

(e.g., Ehrsson 2007; Lenggenhager et al. 2007). "Bodily self-consciousness" is substantially dependent on visual as well as somatosensory cues (Lenggenhager et al. 2009), and tactile input is consulted to build-up a feeling of body ownership (Ehrsson 2012). What is more, tactile input can (amongst other things) be separated (at least to a certain extent) into discriminative and emotional components, mediated by different peripheral nerve fibres (McGlone et al. 2007), indicating that emotional characteristics of tactile input are assessed and differentiated. In sum, one could expect an influence of self-relevance on tactile information processing even stronger than the influence on visual processing. That is, empirical research is consistent with the view that the SPE might vary between the senses.

Assuming differences in the influence of self-relevance according to the sensory modality, it is also reasonable to consider that the SPE may be absent outside vision, since the same holds for other phenomena than self-prioritization when compared between the senses (see, e.g., Barsalou et al. 2003). Note that the assessment of self-relevance effects in the matching paradigm is characterized by the assignment of formerly neutral stimuli to particular labels. Remarkably, these neutral stimuli have up to now always been presented visually, resulting in a to-be-learned combination of two visually presented stimuli (e.g., a shape and a label). Even the extension of the SPE to movements implied the combination of visually presented labels and visual equivalents of the movements (i.e., the mouse cursor following the participant's movement). In the present study, the matching paradigm was implemented with the to-be-associated neutral stimuli presented to sensory modalities other than vision (and with no visual component or counterpart). Importantly, in the field of self-experience, multisensory integration is stated to be a necessary condition for self-identification and discrepancies between the pieces of information reaching different sensory modalities are postulated to reduce the experience of ownership (Tsakiris 2012). Assuming that sensory integration and overlap are required for self-referential processing, possibly the combination 
of a (visually presented) label and a visually presented stimulus is necessary to find effects on

associative learning. Hence, the association of visually presented labels with auditory or tactile stimuli might be unaffected by self-relevance, because the sensory overlap between the stimuli is missing. That is, it is possible that no SPE would be found with auditory or tactile neutral stimuli in the matching paradigm used previously with just visual stimuli.

In addition, whereas visual stimuli may occur in the periphery, self-relevant tactile input is always spatially close to the own body and might therefore per se be judged as selfrelevant. That is, studies on self-experience and on bodily illusions typically do not deal with externally-presented stimuli. Thus, since tactile stimulation always occurs close to the bodily self, it seems plausible that there is no differentiation between self- and other-relevant inputs in touch (though one might consider the fact that the sources causing the tactile stimulation must not obligatory be close, too). Accordingly, in a study designed to assess people's identification of their own hand, participants failed to recognize their own hand from amongst other objects if they were not explicitly informed that they might see their own hand (Wuillemin and Richardson 1982). Hence, again, one may argue that the SPE should be absent in the tactile modality.

Importantly, however, the extension of the SPE from visual perception to action might be interpreted to indicate that self-prioritization represents a general effect. After all, effects of self-relevance in different sensory modalities have often been observed in the literature prior to the introduction of the matching task to assess self-prioritization. For example, the socalled "Cocktail-party effect" was first observed in the dichotic listening task (Moray 1959; see Conway et al. 2001, for the influence of working-memory capacity), indicating that auditory stimuli presented from an ignored location/“channel” can attract attention if they happen to be self-relevant (see also Bargh 1982). Additionally, magnetoencephalographic examinations of the auditory cortex have revealed that self-triggered sounds are distinguished from externally triggered sounds, indicating a neuronal separation (Martikainen et al. 2005). 
Thus, assuming that the SPE represents effects of self-relevance as measured independently of

familiarity (which does not hold for previous self-relevant studies using, e.g., the participant's name, or picture, as self-relevant stimuli), then it should equally emerge for visual, auditory, and tactile stimuli.

When introducing the matching paradigm, Sui and her colleagues suggested that the biased competition model of attention (Desimone and Duncan 1995; Duncan et al. 1997) might underlie the SPE. In brief, the idea is that sensory input in the primary (visual) cortex can be boosted by top-down influences. Self-relevance - even if only recently and arbitrarily acquired - can be seen as such a top-down influence, i.e., the default-value of the cognitive system is to prioritize self-relevant information at quite early stages of (visual) processing. This mechanism might well operate for the other sensory modalities. Based on this notion, together with the assumption that the SPE in the matching paradigm reflects previous effects of self-relevance, it was hypothesized that top-down influences can also boost self-relevant information in senses other than just vision. Such a result would support the claim that this "self-relevance boost" reflects a general and modality-independent mechanism. In this sense, we predict that the SPE should emerge in different sensory modalities.

In order to explore the hypothesis that a general, modality-independent component of the self-prioritization process exists, we adapted Sui and colleagues' (2012) paradigm in such a way that formerly neutral sounds or vibrotactile rhythms, rather than visually-presented geometric forms, were associated with the participant's self, a familiar person, and a neutral stimulus. Investigating the SPE for audition and touch and comparing it between the senses, the current study aimed to provide insights on self-relevant information processing irrespective of the modality.

\section{Overview}


In Experiment 1, we assessed the visual SPE using an adapted version of the selfprioritization paradigm (cf. Sui et al. 2012). That is, participants learned the mapping of three geometric shapes to the self (i.e., the label "I") vs. to two other-relevant labels (i.e., the labels "mother" and "chair") in a sequential learning phase. After this learning phase, the participants were presented with one of the stimuli, followed by one of the labels. Their task was to indicate whether or not the stimulus-label combinations matched the association rules that had been learned previously (matching task). Experiment 2 constituted a replication of Experiment 1, with the only difference being that neutral sounds were now used instead of geometric shapes. Meanwhile, in Experiment 3, vibrotactile rhythms were used as stimuli. Experiments 2 and 3 enabled us to test whether the SPE - reflecting a pure measure of selfprioritization apart from familiarity - also occurs in the auditory and tactile modalities.

In all three experiments, the SPE was indicated by faster and more accurate responses in those trials with the self-associated stimulus (i.e., the stimulus associated with the label "I") compared to responses in trials with one of the other-associated stimuli (i.e., the stimuli associated with the label mother" or "chair"). As in previous research, error rates were analyzed using signal detection indices but mean reaction times (RTs) were the main dependent variable. Note that all three experiments were carried out according to the principles of the Declaration of Helsinki, on the basis of informed consent.

\section{Experiment 1}

To enable direct comparisons of the SPE between the modalities, we used variants of the matching paradigm in which the to-be-associated stimuli were explicitly presented during the learning phase, and with the stimulus-label assignments presented sequentially. This was not the case in previous visual experiments that have used this paradigm in which the participants learned the stimulus-label mappings by means of simultaneously-presented sentences (e.g., see Sui et al. 2012). Hence, Experiment 1 was conducted as a kind of control 
condition whereby three geometric forms, namely a square, a triangle, and a circle, were associated with the labels "I", "mother", and "chair" in a learning phase in which labels and stimuli were explicitly presented.

\section{Methods}

Participants. Thirty students (26 female) with a median age of 22 years (ranging from 18 to 29 years) participated, all having normal or corrected-to-normal vision. The mean error rate of two participants and the average RT of three participants constituted an outlier according to Tukey (1977) when compared to the sample distribution of the mean error rates or average RTs of all participants. The data from these five participants were therefore excluded from the data analyses.

The SPEs were rather large in previous studies $(d z>0.81$ in Sui et al. 2012). Given $N=$ $25, \alpha=.05$, and a power of $1-\beta=.80$, an effect of size $d z \geq 0.58$ could be detected (G*Power

\subsection{3, Faul et al. 2007).}

Design. The experiment consisted of a 3 (visual stimulus: self-associated vs. motherassociated vs. chair-associated) $\times 2$ (matching condition: matching vs. non-matching) withinparticipants design.

Apparatus and materials. The experiment was conducted on PCs with 24''-TFT screens using standard computer mice. The experiment was run by E-Prime 2.0 software. The stimuli consisted of visual geometric shapes. The labels used were the German word "Ich" $[\mathrm{I}]$ as a self-relevant stimulus, the German word "Mutter" [mother] as a familiar stimulus, and the German word "Stuhl” [chair] as a neutral stimulus. These words were presented in white against a black background and in Courier New typeface at a viewing distance of $50 \mathrm{~cm}$, resulting in a visual angle of about $0.7^{\circ}$. In the matching-task trials, the geometric shapes, the labels, and a fixation cross at the start of each trial were presented from the center of the computer screen, subtending $5.7^{\circ} \times 5.7^{\circ}$ visual angle for the geometric shapes. The assignment 
of the geometric shapes to the labels was balanced across participants following a Latinsquare design.

Procedure. Participants were tested in sound-proofed rooms. The task instructions were briefly summarized by the experimenter and were also presented on the screen; responses involved presses of the right and left button of the computer mouse.

At the beginning of the experimental session, the participants learned the assignments of the labels (I vs. mother vs. chair) to the geometric shapes (triangle vs. square vs. circle). The participants had to remember the stimulus-label assignments and were told that they had to evaluate stimulus-label pairings with respect to this assignment in the second phase of the study. A trial of the learning phase started with the presentation of a label. After $500 \mathrm{~ms}$ presenting the label only, the to-be-associated stimulus was presented for 2,500 ms (with the label still visible) and followed by a blank screen for 2,000 ms. Each stimulus-label pairing was presented four times during the learning phase, resulting in a total of 12 trials.

Closely following Sui and colleagues' (2012) procedure, the participants next went through a practice phase of the matching task. Therefore, participants were instructed to place their right index finger on the left mouse button (matching response) and their right middle finger on the right mouse button (non-matching response). Each trial of the practice phase started with the presentation of a black screen $(500 \mathrm{~ms})$, followed by a fixation cross (again $500 \mathrm{~ms}$ ). The visual stimulus was presented for $500 \mathrm{~ms}$ and followed by the label which remained on the screen until the participant responded. At the end of each trial, participants received feedback whether their response had been correct or not.

After 12 practice trials (six matching and six non-matching trials), the participants started the experimental phase. Again, their task was to judge whether the displayed stimuluslabel pairing corresponded to the initially learned assignment (press the left mouse button) or not (press the right mouse button). The participants were only provided with feedback when their response was incorrect. The experimental phase consisted of 192 trials presented in 
random order. In detail, each label was presented in 64 trials, half of them with matching pairings and half of them with non-matching pairings, resulting in 32 matching and 32 nonmatching trials for each label.

\section{Results}

For all of the analyses reported in Experiments 1-3, only correct responses with RTs above $100 \mathrm{~ms}$ and below three interquartile ranges above the third quartile of the overall distribution of correct RTs (Tukey 1977) were used for the RT analysis. Further, for all of the statistical analyses reported here, a significance level of $\alpha=.05$ was specified. In Experiment 1, averaged across participants, $96.2 \%$ of the trials were selected for RT analysis; $3.1 \%$ of the trials were excluded because of erroneous responses and $0.8 \%$ due to the RT outlier criteria.

Average RTs. The RT data were submitted to a 3 (visual stimulus: self- vs. mother-vs. chair-associated $) \times 2$ (matching condition: matching vs. non-matching) within-participants MANOVA (see O'Brien and Kaiser 1985, for the use of MANOVA to analyze repeatedmeasures designs). The main effects of visual stimulus, $F(2,23)=10.68, p=.001, \eta_{\mathrm{p}}{ }^{2}=.48$, and matching condition, $F(1,24)=27.97, p<.001, \eta_{\mathrm{p}}{ }^{2}=.54$, were both significant. Additionally, there was a significant visual stimulus $\times$ matching condition interaction, $F(2$, 23) $=6.24, p=.007, \eta_{\mathrm{p}}^{2}=.35$.

To probe the interaction of label and matching condition and following Sui and colleagues' logic according to the SPE, we analyzed matching and non-matching trials separately (see Figure 1a). As mentioned above, the effect was indicated by faster responses in matching trials with the self-associated stimulus (i.e., the stimulus associated with the label "I") compared to responses in matching trials with one of the other-associated stimuli (i.e., the stimuli associated with the label mother" or "chair"), which was reflected by a Helmert contrast. In a one-factorial MANOVA with matching trails and the within-participant factor of 
visual stimulus revealed a significant Helmert contrast, $F(1,24)=22.87, p<.001, \eta_{\mathrm{p}}{ }^{2}=.49$

$(d z=0.96)$, indicating a significant SPE in the RT data (see Figure 1a). In non-matching trials, this Helmert contrast was also significant, $F(1,24)=12.86, p=.001, \eta_{\mathrm{p}}{ }^{2}=.35$, indicating that even in non-matching trials RTs depended on the stimulus association. -- insert Fig1 about here --

Fig 1 Mean RTs as a function of the particular stimulus (self- vs. mother- vs. chairassociated) and matching condition (matching vs. non-matching) (a) with visual stimuli (Experiment 1), (b) with auditory stimuli (Experiment 2), and (c) with tactile stimuli (Experiment 3). The error bars indicate the standard errors of the means.

Table 1 Error rates (in \%) as a function of matching condition (matching vs. non-matching), stimulus association (self-associated vs. mother-associated vs. chair-associated), and sensory modality (vision vs. audition vs. touch). Standard deviations in brackets.

\section{Matching Condition}

$\overline{\text { Matching Non-matching }}$

Stimulus association

\begin{tabular}{ccccccc}
$\begin{array}{c}\text { Sensory } \\
\text { Modality }\end{array}$ & Self & Mother & Chair & Self & Mother & Chair \\
\hline $\begin{array}{c}\text { Vision } \\
(\text { Exp. 1) }\end{array}$ & $2.1(2.8)$ & $4.6(3.6)$ & $4.3(5.7)$ & $1.8(2.9)$ & $3.6(3.5)$ & $2.1(2.8)$ \\
$\begin{array}{c}\text { Audition } \\
(\text { Exp. 2) }\end{array}$ & $6.7(8.9)$ & $8.5(6.5)$ & $9.4(8.3)$ & $6.3(5.8)$ & $9.0(8.1)$ & $7.8(6.0)$ \\
$\begin{array}{c}\text { Touch } \\
(\text { Exp. 3) }\end{array}$ & $4.0(5.3)$ & $3.9(6.5)$ & $6.0(5.6)$ & $4.9(6.8)$ & $4.3(4.9)$ & $4.7(5.5)$
\end{tabular}


Sensitivity measures. Mean error rates for Experiment 1-3 are shown in Table 1. The

error data were analyzed using signal-detection sensitivity indices for each stimulus condition (cf. Sui et al. 2012). To this end, we considered correct responses in matching trials to represent hits and erroneous responses in matching trials to represent misses. Correct responses in non-matching trials were considered correct rejections and erroneous responses were treated as false alarms. Following the so-called log-linear approach to account for cases with $100 \%$ hits or $0 \%$ false alarms (see Hautus 1995; Stanislaw and Todorov 1999), we then computed $d$ ' as a measure of sensitivity. That is, we added 0.5 to the number of hits and the number of false alarms and we added 1 to the number of signal trials and the number of noise trials before calculating the hit and false alarm rates.

The resulting $d$ ' indices were submitted to a one-factorial MANOVA with 3 levels (visual stimulus: self- vs. mother- vs. chair-associated). The relevant Helmert contrast was significant, $F(1,24)=11.50, p=.002, \eta_{\mathrm{p}}{ }^{2}=.32$, indicating a significant SPE in sensitivity measures.

\section{Experiment 2}

In Experiment 2, the participants learned to associate three different auditory stimuli to the labels "I", "mother", and "chair". That is, for the first time, auditory stimuli were used to test whether or not there is an auditory SPE for formerly neutral stimuli that became associated with the self. The design and procedure were the same as in Experiment 1 with the only exceptions concerning small adaptations of the experimental set-up and stimulus presentation resulting from the change of the stimulus modality.

\section{Methods}

Participants. Thirty-three volunteers (20 female) were recruited at the University of Trier in exchange for course credit. Their median age was 22 years (ranging from 18 to 27 years) and all participants had normal or corrected-to-normal vision. The mean error rate of 
two participants constituted an outlier according to Tukey (1977) when compared to the

sample distribution of the mean error rates of all participants. The data from these participants were therefore excluded from the data analyses.

Given $N=31, \alpha=.05$, and a power of $1-\beta=.80$, an effect of size $d z \geq 0.52$ could be detected (G*Power 3.1.3, Faul et al. 2007).

Apparatus and materials. The apparatus was the same as in Experiment 1 with the exception that auditory stimuli served as stimuli. The stimuli consisted of instrumental tones as generated by a flute, a snare drum, and a woodblock (percussion instrument). In a pilot study, these sounds had been rated to be equal in valence (non-significant difference from neutral on a 9-point Likert-scale with all $p \mathrm{~s} \geq .190 ; M_{\text {flute }}=4.92[S D=1.62] ; M_{\text {snare drum }}=4.93$ $\left.[S D=0.93] ; M_{\text {woodblock }}=4.65[S D=1.43]\right)$. The auditory stimuli on a given trial had an intensity of approximately $50 \mathrm{~dB}$ SPL and were presented for $300 \mathrm{~ms}$ via headphones. In the matching-task trials, the fixation cross presented at the start of each trial as well as the label were both presented $21.0^{\circ}$ from the upper border of the screen.

Procedure. The procedure was essentially the same as in Experiment 1. Yet, in the learning phase, after presenting the label for $500 \mathrm{~ms}$, the to-be-associated stimulus was presented for $300 \mathrm{~ms}$ (with the label still visible) and after the presentation of the sound, the label remained on the screen for another $2,200 \mathrm{~ms}$, resulting in a total label-presentation time of $3,000 \mathrm{~ms}$.

\section{Results}

Averaged across participants, $92.1 \%$ of the trials were selected for RT analysis; $7.9 \%$ of the trials were excluded because of erroneous responses and $0 \%$ due to the RT outlier criteria.

Average RTs. In a 3 (auditory stimulus: self-vs. mother-vs. chair-associated) $\times 2$ (matching condition: matching vs. non-matching) within-participants MANOVA, the main 
effect of auditory stimulus just missed the conventional criterion of significance, $F(2,29)=$

$3.15, p=.058, \eta_{\mathrm{p}}^{2}=.18$. The main effect of matching condition was significant, $F(1,30)=$ 40.62, $p<.001, \eta_{\mathrm{p}}^{2}=.58$ and, importantly, the interaction between the factors of auditory stimulus and matching condition was significant too, $F(2,29)=3.46, p=.045, \eta_{\mathrm{p}}{ }^{2}=.19$, thus suggesting that the RT differences for the labels differed for matching and non-matching trials.

Analyzing matching and non-matching trials separately, a one-factorial MANOVA with matching trials and the within-participant factor of auditory stimulus (self-vs. mothervs. chair-associated) revealed a significant Helmert contrast, $F(1,30)=8.16, p=.008, \eta_{\mathrm{p}}{ }^{2}=$ $.21(d z=0.51)$, meaning that RTs were shorter in self-relevant matching trails than in otherrelevant matching trials. In non-matching trials, this Helmert contrast was not significant, $F(1$, $30)=2.17, p=.151, \eta_{\mathrm{p}}{ }^{2}=.07$, showing that RTs in non-matching trials were not affected by the association of the stimuli.

Sensitivity measures. Once again, error rates were analyzed using signal detection sensitivity indices for each label condition. A one-factorial MANOVA with 3 levels (auditory stimulus: self- vs. mother- vs. chair-associated) yielded a significant Helmert contrast, $F(1$, $30)=5.41, p=.027, \eta_{\mathrm{p}}^{2}=.15$, indicating a higher sensitivity in trials with the self-relevant stimulus than in trials with other-relevant stimuli.

\section{Experiment 3}

In Experiment 3, we assessed the SPE in touch. That is, three different vibrotactile rhythms were now associated with the labels "I", "mother", and "chair". The design and procedure were essentially the same as those in Experiments 1 and 2.

\section{Methods}

Participants. Twenty-four students (22 female) at the University of Trier participated in the experiment in exchange for course credit. Their median age was 21 years (ranging from 
18 to 25 years) and all participants had normal or corrected-to-normal vision. Given $N=24, \alpha$

$=.05$, and a power of $1-\beta=.80$, an effect of size $d z \geq 0.60$ could be detected (G*Power 3.1.3,

Faul et al. 2007). Note that the SPEs in Sui et al. (2012) were $d z>0.81$.

Apparatus and materials. The apparatus was the same as in Experiments 1 and 2.

Additionally, in this experiment, E-Prime controlled the presentation of the vibrotactile stimuli via a serial interface. That is, the vibrotactile stimuli were delivered by means of a tactor (Model C-2, Engineering Acoustic, Inc.) which was driven independently from the PC by an individual standard amplifier (Power Amplifier Module PM40C, t.amp). This tactor was $1.17^{\prime \prime}$ in diameter and 0.30 ' thick and it was fastened to the side of the participant's left palm by means of a Velcro strip.

Three vibrotactile stimuli with different rhythms were used throughout the experiment (see Figure 2, for a schematic illustration). The first vibration comprised two 150-ms pulses separated by a 200-ms pause (Stimulus A). The second vibration consisted of a continuous 500-ms pulse (Stimulus B) and the third vibration consisted of a 150-ms pulse followed by three 50-ms pulses, whereby each of the four pulses was followed by a 50-ms pause (Stimulus C). The tactile stimuli on a given trial were presented for $500 \mathrm{~ms}$.

-- insert Fig2 about here --

Fig 2 Schematic illustration of the three vibrotactile stimuli, each consisting of ten 50-ms intervals. Vibrations are indicated by grey intervals, pauses by white intervals

Procedure. In order to reduce the amount of environmental noise to a minimum, participants were tested in a completely light- and soundproofed room. They had their left hand positioned at their body midline (i.e., in the direction where the head was oriented). To limit and control for any potential auditory cues emitted by the operation of the transducers, the participants' hands were lifted up by placing their left wrist on an armrest. Additionally, white noise was presented via headphones to rule out any influence of auditory signals (e.g., 
those attributable to the activation of the transducers). In each trial a black screen (500 ms), a

fixation cross $(500 \mathrm{~ms})$, a tactile stimulus $(500 \mathrm{~ms})$, another fixation cross $(50 \mathrm{~ms})$, and a label were sequentially presented. The label remained on the screen until the participant responded.

\section{Results}

Averaged across participants, $94.3 \%$ of the trials were selected for RT analysis; $4.6 \%$ of the trials were excluded because of erroneous responses and $1.1 \%$ due to the RT outlier criteria.

Average RTs. A 3 (vibrotactile stimulus: self-vs. mother-vs. chair-associated) $\times 2$ (matching condition: matching vs. non-matching) within-participants MANOVA revealed a non-significant main effect of the vibrotactile stimulus, $F(2,22)=2.80, p=.083, \eta_{\mathrm{p}}{ }^{2}=.20$. Nevertheless, a significant main effect of matching condition was observed, $F(1,23)=50.95$, $p<.001, \eta_{\mathrm{p}}^{2}=.69$, which was qualified by a significant interaction with the vibrotactile stimulus factor, $F(2,22)=4.60, p=.021, \eta_{\mathrm{p}}{ }^{2}=.30$. Analyzing matching and non-matching trials separately, a one-factorial MANOVA with matching trials and the within-participant factor of vibrotactile stimulus revealed a significant Helmert contrast (self-relevant vs. otherrelevant matching trials), $F(1,23)=9.44, p=.005, \eta_{\mathrm{p}}{ }^{2}=.29(d z=0.64)$, reflecting a significant SPE in the RT data (see Figure 1b). In non-matching trials, this Helmert contrast was non-significant, $F<1$, thus revealing that RTs in non-matching trials were not affected by the association of the stimuli to self- or other-relevant labels.

Sensitivity measures. To analyze the error rates, sensitivity indices $d$ ' were again submitted to a one-factorial MANOVA with 3 levels (vibrotactile stimulus: self- vs. mothervs. chair-associated). The Helmert contrast was not significant, $F<1$, indicating no significant SPE in sensitivity measures.

\section{Comparison of the SPE across modalities}


To compare the size of the SPE in vision, audition, and touch, the data of all participants included in the analyses of Experiments 1-3 were submitted to a one-factorial ANOVA with the between-participants factor of stimulus modality (vision vs. audition vs. touch). As the dependent variable, the SPE was computed by subtracting the mean RT/d' in trials with the self-associated stimulus from the average RT/d' in trials with one of the other-associated stimuli. We conducted one ANOVA for SPEs based on RTs and the same ANOVA for SPEs based on $d^{\prime}$. To control for processing differences between the senses, we adjusted each participant's SPE, dividing it by his/her mean RT or his/her mean $d^{\prime 1}$. With regard to the RT data, the ANOVA revealed no significant main effect of stimulus modality (vision vs. audition vs. touch), $F(2,77)=1.66, p=.198, \eta_{\mathrm{p}}{ }^{2}=.04$, indicating that the adjusted RT-based SPE did not differ significantly between the senses ${ }^{2}$. With regard to the sensitivity measures, the adjusted $d$ '-based SPE did also not differ significantly between the stimulus modalities, $F$ $<1$. Taken together, these analyses suggest that modality did not moderate the SPE.

To provide further support for this conclusion, we calculated Bayes factors using the Bayes-ANOVA module of JASP (Love et al. 2015). The Bayes factor in favor of the null model is $\mathrm{BF}_{01}=2.55$ for the adjusted RT-based $\mathrm{SPE}$ and $\mathrm{BF}_{01}=5.18$ for the adjusted $d$ '-based SPE. According to the rules-of-thumb given by Raftery (1995), the former value is considered "weak" evidence for the null hypothesis whereas the latter is categorized as "positive" evidence.

Moreover, to approach the question of whether the processes underlying the SPE differ between vision, audition, and touch, we analyzed the RT-based SPE's dependence on the duration of mean RTs by a distribution analysis (delta plots). Therefore, mean RTs of correct

\footnotetext{
${ }^{1}$ For all three stimulus modality conditions, the analyses with the adjusted SPEs replicated the reported effects (in RTs: $t(24)=5.18, p<.001, d=1.04$, for vision, $t(30)=3.25, p=.003, d=0.58$, for audition, and $t(23)=$ $3.25, p=.004, d=0.66$, for touch; in $d^{\prime}: t(24)=-3.43, p=.002, d=0.69$, for vision, $t(30)=-2.01, p=.027$ (onetailed), $d=0.36$, for audition, and $t(23)=-0.58, p=.571, d=0.12$, for touch).

${ }^{2}$ Note that the power to detect a large effect $(f=0.4)$ between the stimulus modalities, given an $\alpha$-value of .05 and $N=80$, was $1-\beta=.89$ (calculations performed with $\mathrm{G}^{*}$ Power 3.1.3; Faul et al. 2007).
} 
responses were vincentized (Ratcliff 1979) separately for each of the conditions of the 3

(stimulus: self- vs. mother- vs. chair-associated $) \times 2$ (matching condition: matching vs. nonmatching) design. Individual mean RTs were derived for each quintile of the rank-ordered raw RT data. To reduce complexity, we used the RT-based SPE (rather than mean RTs for each quintile and condition) as the dependent variable. A 5 (quintile) $\times 3$ (stimulus modality: vision vs. audition vs. touch) MANOVA for repeated measures with SPE as the dependent variable yielded a significant main effect of quintile, $F(4,74)=8.97, p<.001, \eta_{\mathrm{p}}^{2}=.327$, which was not moderated by stimulus modality, $F<1$. Only the linear trend was significant, $F(1,77)=17.93, p<.001, \eta_{\mathrm{p}}^{2}=.189(F<1.16$ for the moderation by modality $)$. This indicates that the SPE increased significantly with the duration of mean RTs (a pattern routinely expected for RT effects; e.g., Schwarz and Miller 2012) and moreover that this increase is independent of the stimulus modality.

\section{General Discussion}

In the present study, we investigated the SPE using a matching task in vision, audition, and touch (Experiments 1-3, respectively). Specifically, we compared performance in those trials with a stimulus that had been associated with the self to performance in trials with otherassociated stimuli. In all three experiments, as hypothesized, performance in self-associated trials was faster than performance in other-associated trials. That is, the typical pattern of data was replicated for the visual modality and it was, for the first time, demonstrated in the auditory and tactile modalities as well. Note that the learning phase in all three experiments reported here was slightly different from that commonly used in the matching paradigm. That is, the to-be-associated stimuli were not introduced semantically (i.e., presenting the word "triangle"), but directly presented (i.e., presenting a triangle) and the shape-label associations were presented sequentially in random order in the learning phase. Still, in line with previous research, a prioritization of self-relevant pairings occurred irrespective of the stimulus 
modality. Furthermore, the results are in line with the assumption of top-down influences on

information processing in the visual cortex as stated by the biased competition model of attention (Desimone and Duncan 1995). Additionally, the results may reflect similar modulatory top-down influences on the sensory input in auditory and somatosensory cortex.

Taken together, the finding of comparably-sized SPEs in the different senses suggests that self-prioritization represents a general top-down influence on sensory processing. This is in line with previous research highlighting the importance of self-relevance in different sensory modalities, namely audition (e.g., the "Cocktail-party effect”; Moray, 1959) and touch (e.g., the full-body illusion; Ehrsson 2007; Lenggenhager et al. 2007). Yet, previous findings in different modalities did not use the matching paradigm to assess self-prioritization independently from familiarity of the stimulus materials. Rather, the participant's own name was, for example, often used as the self-relevant stimulus. In contrast, the matching paradigm represents a tool for measuring self-relevance independently from familiarity, because here formerly neutral stimuli become associated to the self vs. others throughout the experimental procedure. Hence, the supramodal observation of self-prioritization in the current experiments reflects original evidence for a general tendency to prioritize arbitrarily-tagged self-relevant contents independently of familiarity. This strengthens the conclusion that self-relevance represents an important mechanism in selection as to guide human behavior in a perceptually complex environment. The top-down component of the SPE in the matching task has previously been interpreted to directly impact sensory input. For example, an interaction of the effect with visual degradation, assumed to modulate early visual processing (Sui et al. 2012), and postulated neuronal as well as behavioral parallels of self-relevance and perceptual saliency (Sui et al. 2013a) support this hypothesis. Nevertheless, other findings suggest that the SPE may not only affect the sensory input but potentially later stages of information processing as well. For example, a memory advantage for self-related pairings was shown, indicating a better individuation of self-relevant associations rather than a perceptual benefit 
(Fuentes et al. 2015) and a prioritization of abstract concepts rather than specific percepts

emphasizes conceptual components of self-prioritization (Schäfer et al. 2015).

There are some methodological drawbacks with regard to the current study that should be mentioned. First, significant SPEs in all three experiments were found in the RT data but not in the sensitivity indices. In the sensitivity data, significant self-prioritization was observed in audition (Experiment 2) and vision (Experiment 1). Yet, no significant SPE was documented in touch (Experiment 3). Still, importantly, there was no evidence for any speed accuracy trade-off. This absence of a SPE with regard to the sensitivity may be related to the consistently high accuracy (indicated by low error rates in all conditions). Second, the power to detect an effect of the sensory modalities on the size of the SPE was only acceptable for large effect sizes in the current study (i.e., if there are modality-specific effects on the SPE with middle or low effect sizes, we could have missed them due to power issues). Yet, the conclusion that the SPE is independent of the sensory modalities is further supported by another statistical approach (namely the Bayesian approach). Nonetheless, our results clearly show that self-prioritization influences information processing in different sensory modalities.

Overall, the current approach and results open the door to a number of intriguing questions regarding self-prioritization processes. For example, the RT distribution analysis revealed that the increase of the SPE with mean RTs was not modulated by the sensory modality. This provides only indicative evidence that the basic processes causing the SPE are amodal so that the results cannot unequivocally be taken to imply that self-prioritization is attributable to the same processes in all (tested) sensory modalities. As the current experiment was not conducted to investigate the contribution of specific cognitive processes to the SPE, future research is needed in order to test whether the SPE is modulated by the same factors in all three sensory modalities (e.g., whether the degradation of sensory input bolsters the SPE in audition and touch in the same way as it has been observed for vision). 
In addition, the locus of the SPE needs further investigation. In particular, Schäfer and her colleagues (2015) demonstrated that an abstract representation of a stimulus is mapped onto the to-be-associated label. Taken together with the findings of the current research, this raises the question of whether the association of a stimulus to the self leads to its prioritization on a supramodal level. To test this question, future research may use a crossmodal variant of the matching task.

In summary, the behavioral data obtained in the present study extend the finding that self-prioritization influences human information processing from the visual modality to the sensory modalities of audition and touch. That is, we present the first study to show that auditory and tactile stimuli that become associated with the self (albeit arbitrarily) are prioritized when compared to other-associated stimuli within the same modality. In conclusion, self-prioritization represents a top-down mechanism influencing the processing of sensory input across vision, audition, and touch. 


\section{Conflict of interest}

The authors declare that they have no conflict of interest. 


\section{REFERENCES}

Alexopoulos T, Muller D, Ric F, Marendaz C (2012) I, me, mine: Automatic attentional capture by self-related stimuli. European Journal of Social Psychology 42:770-779 doi: 10.1002/ejsp. 1882

Bargh JA (1982) Attention and automaticity in the processing of self-relevant information. Journal of Personality and Social Psychology 43:425-436 doi: 10.1037/00223514.43.3.425

Barsalou LW, Simmons WK, Barbey AK, Wilson CD (2003) Grounding conceptual knowledge in modality-specific systems. Trends in Cognitive Sciences 7:84-91. doi: 10.1016/S1364-6613(02)00029-3

Conway ARA, Cowan N, Bunting, MF (2001) The cocktail party phenomenon revisited: The importance of working memory capacity. Psychonomic Bulletin \& Review 8:331-335. doi: 10.3758/BF03196169

Desimone R, Duncan J (1995) Neural mechanisms of selective visual attention. Annual Review of Neuroscience 18:193-222 doi: 10.1146/annurev.ne.18.030195.001205

Duncan J, Humphreys G, Ward R (1997) Competitive brain activity in visual attention. Current Opinion in Neurobiology 7:255-261 doi: 10.1016/S0959-4388(97)80014-1

Ehrsson HH (2007) The experimental induction of out-of-body experiences. Science 317:1048 doi: $10.1126 /$ science. 1142175

Ehrsson HH (2012) The concept of body ownership and its relation to multisensory integration. In: Stein BE (ed) The New Handbook of Multisensory Processes. MIT Press, Cambridge, MA, pp 775-792

Faul F, Erdfelder E, Lang A-G, Buchner A (2007) GPower 3: A flexible statistical power analysis program for the social, behavioral and biomedical sciences. Behavioral Research Methods 39:175-191 doi: 10.3758/BF03193146 
Frings C, Wentura D (2014) Self-priorization processes in action and perception. Journal of Experimental Psychology: Human Perception and Performance 40:1737-1740 doi: $10.1037 / \mathrm{a} 0037376$

Fuentes LJ, Sui J, Estévez AF, Humphreys GW (2015) The differential outcomes procedure can overcome self-bias in perceptual matching. Psychonomic Bulletin \& Review:1-8 doi: $10.3758 / \mathrm{s} 13423-015-0895-3$

Gallace A, Spence C (2014) In touch with the future: The sense of touch from cognitive neuroscience to virtual reality. Oxford University Press, Oxford, UK

Giesbrecht B, Sy JL, Lewis MK (2009) Personal names do not always survive the attentional blink: Behavioral evidence for a flexible locus of selection. Vision Research 49:13781388 doi: 10.1016/j.visres.2008.02.013

Hautus MJ (1995) Corrections for extreme proportions and their biasing effects on estimated values of d'. Behavior Research Methods, Instruments \& Computers 27:46-51 doi: $10.3758 / \mathrm{BF} 03203619$

Lenggenhager B, Mouthon M, Blanke O (2009) Spatial aspects of bodily self-consciousness. Consciousness and Cognition 18:110-117 doi: 10.1016/j.concog.2008.11.003

Lenggenhager B, Tadi T, Metzinger T, Blanke O (2007) Video ergo sum: Manipulating bodily self-consciousness. Science 317:1096-1099 doi: 10.1126/science.1143439

Love J, Selker R, Marsman M, Jamil T, Dropmann D, Verhagen AJ, Ly A, Gronau QF, Smira M, Epskamp S, Matzke D, Wild A, Rouder JN, Morey RD, Wagenmakers EJ (2015) JASP (Version 0.7) [Computer software].

Martikainen MH, Kaneko K-i, Hari R (2005) Suppressed responses to self-triggered sounds in the human auditory cortex. Cerebral Cortex 15:299-302 doi: 10.1093/cercor/bhh131

McGlone F, Vallbo AB, Olausson H, Loken L, Wessberg J (2007) Discriminative touch and emotional touch. Canadian Journal of Experimental Psychology 61:173-183 doi: 10.1037/cjep2007019 
Moray N (1959) Attention in dichotic listening: Affective cues and the influence of instructions. Quarterly Journal of Experimental Psychology 11:56-60 doi: $10.1080 / 17470215908416289$

O'Brien RG, Kaiser MK (1985) MANOVA method for analyzing repeated measures designs: An extensive primer. Psychological Bulletin 97:316-333 doi: 10.1037/00332909.97.2.316

Raftery AE (1995) Bayesian model selection in social research. Sociological Methodology 25:111-163

Ratcliff R (1979) Group reaction time distributions and an analysis of distribution statistics. Psychological Bulletin 86:446-461

Schäfer S, Wentura D, Frings C (2015) Self-prioritization beyond perception. Experimental Psychology 62:415-425

Schwarz W, Miller J (2012) Response time models of delta plots with negative-going slopes. Psychonomic Bulletin \& Review, 19:555-574

Stanislaw H, Todorov N (1999) Calculation of signal detection theory measures. Behavior Research Methods, Instruments \& Computers 31:137-149 doi: 10.3758/BF03207704

Sui J, He X, Humphreys GW (2012) Perceptual effects of social salience: Evidence from selfprioritization effects on perceptual matching. Journal of Experimental Psychology: Human Perception and Performance 38:1105-1117 doi: 10.1037/a0029792

Sui J, Liu M, Mevorach C, Humphreys GW (2013a) The salient self: The left intraparietal sulcus responds to social as well as perceptual-salience after self-association. Cerebral Cortex 21:1-9 doi: 10.1093/cercor/bht302

Sui J, Rotshtein P, Humphreys GW (2013b) Coupling social attention to the self forms a network for personal significance. Proceedings of the National Academy of Sciences of the United States of America 19:7607-7612 doi: 10.1073/pnas.1221862110 
Tsakiris M (2010). My body in the brain: A neurocognitive model of body-ownership.

Neuropsychologia 48:703-712. doi: 10.1016/j.neuropsychologia.2009.09.034

Tukey JW (1977) Exploratory data analysis. Reading: Addison-Wesley

Wuillemin D, Richardson B (1982) On the failure to recognize the back of one's own hand.

Perception 11:53-55 doi: 10.1068/p110053

Yang H, Wang F, Nianjun G, Gao X, Zhao G (2013) The cognitive advantage for one's own name is not simply familiarity: An eye-tracking study. Psychonomic Bulletin \& Review 20:1176-1180 doi: 10.3758/s13423-013-0426-z 
a)

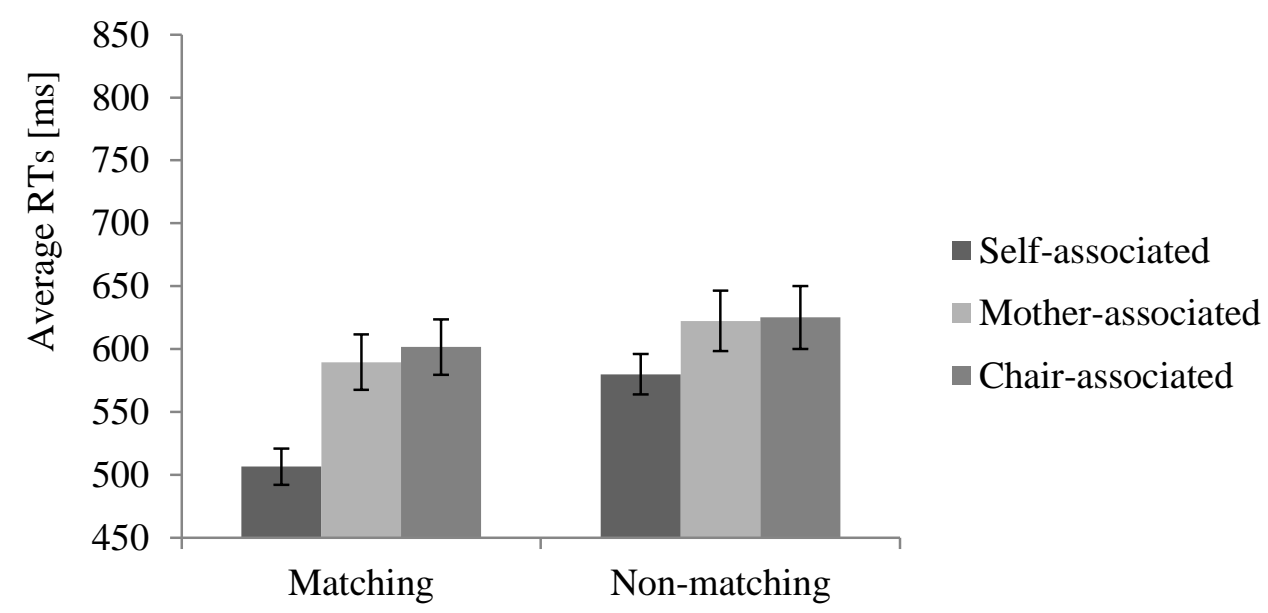

b)

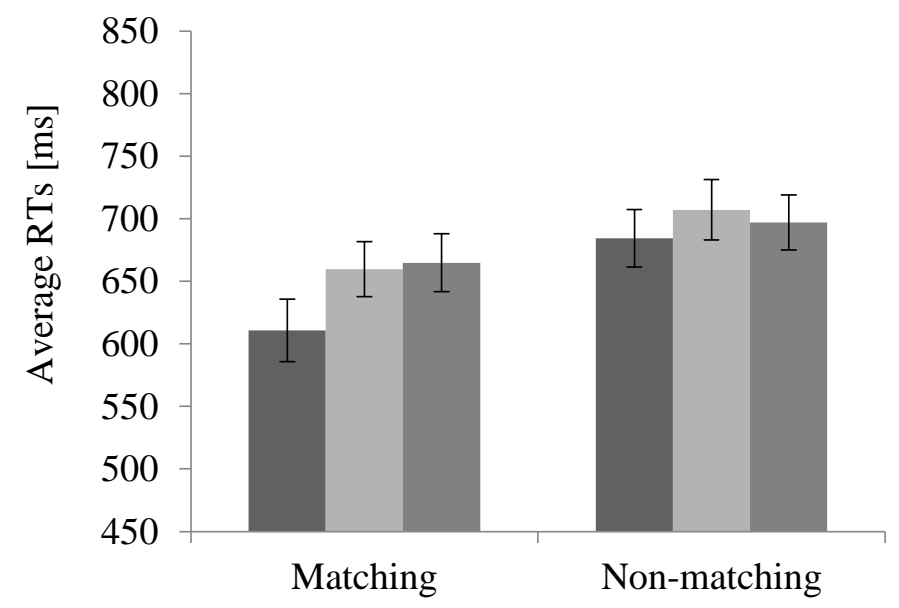

- Self-associated

Mother-associated

Chair-associated

c)

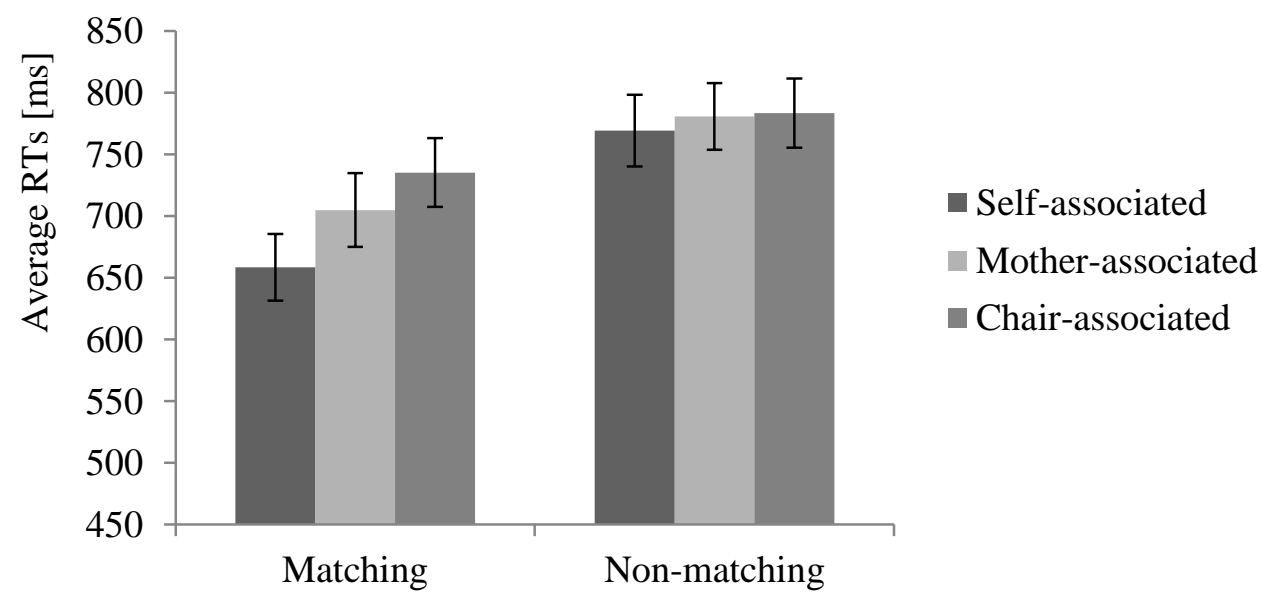


Vibrotactile stimulus A:

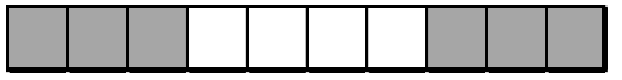

Vibrotactile stimulus B:

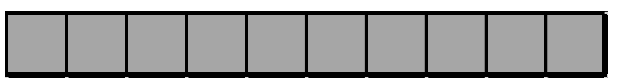

Vibrotactile stimulus C:

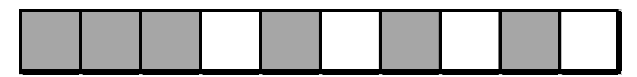

